\title{
ISKO and Knowledge Organization's 25th Anniversary: The Future of Knowledge Organization and ISKO Panel Discussion
}

\author{
Reported by \\ Rebecca Green*
}

*OCLC Online Library Computer Center, Inc.; Dewey Editorial Office, Dewey Section, Library of Congress, 101 Independence Ave. S.E., Washington, DC 20540-4330,

<greenre@oclc.org>

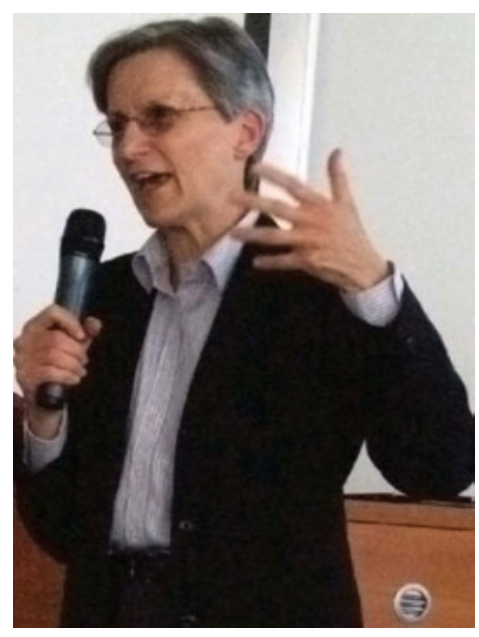

Rebecca Green

The main idea of this panel was to create a platform for discussing knowledge organization in the past, present, and future within ISKO. During the panel discussion the following three questions were asked: 1) What is knowledge organization (KO)? 2) What changes do you foresee in the future that will prove to be the most challenging for ISKO? 3) What is your ideal picture of what the ISKO of the future could be? How do we get there?

\subsection{What is knowledge organization?}

Rebecca Green: I will lead us off with two insights. First, according to the ISKO charter, "It is the aim of the Society [ISKO] to promote research, development and application of all methods for the organization of knowledge in general or of particular fields by integrating especially the conceptual approaches of classification research and artificial intelligence. The Society stresses philosophicological, psychological and semantic approaches for a conceptual order of objects."

Second, in Dewey the rule of application instructs us to class a work on, say, a thesaurus of architecture-that is, the making of a thesaurus applied to architecturewith other works on architecture. But developing a thesaurus on architecture doesn't make the developer an architect. Against that backdrop, what is knowledge organization?

Claudio Gnoli: What is $\mathrm{KO}$ as seen from the perspective of other people? There is a lot of work nowadays that actually is $\mathrm{KO}$ but is called with other names by the com-

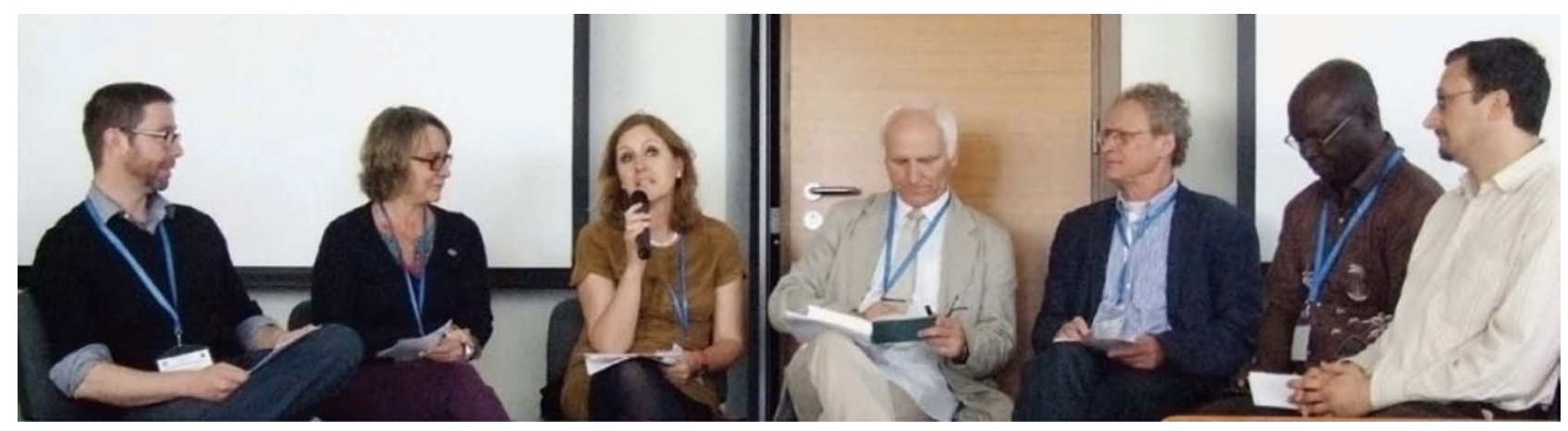

Joseph Tennis, Vera Dodebei, Rosa San Segundo, Wiesław Babik, Peter Ohly, Amos David, Claudio Gnoli. 
munities of, e.g., ontologists, taxonomists, terminologists, topic maps experts, information architects, etc. Unfortunately $\mathrm{KO}$ is often not identified as a field in itself, maybe because it deals with such basic logical components of knowledge (classes, hierarchies, terms, etc.) that people take them for granted.

We as ISKO should include these communities in the discussion, but in order to achieve this we should adopt some common, consistent terminology, quite like Ranganathan did within his own works when writing about devices, canons, etc. I for one am trying to adopt the term dimensions of $\mathrm{KO}$ (that is the ontological, epistemological, pragmatic, etc., dimensions) in the same sense as previously used in Tennis's and Hjørland's papers, although arguing different things.

Ingetraut Dahlberg: When we founded ISKO we needed a new name, other than Society for Classification, since we had just left the German society with this name because it became a society of mathematicians. We thought of Bliss's two books but considered the expression "organization of knowledge" too long in the name of a society and changed it-according to the German way of expressing such combinations - into knowledge organization. To our astonishment, it was accepted worldwide. However, we just meant it as another name for order activities in classification. Our journal International Classification had a current bibliography of relevant titles. The classification system for this bibliography remained the same when we renamed the journal Knowledge Organization. I referred to this and explained its structure in my articles of KO 2006 and 2014. I consider knowledge organization as a subdiscipline of the science of science with application fields not only in the information sciences but also for all subject fields (domains) needing taxonomies (classification systems of objects) and other fields like statistics, commodities, utilities, weapons, patents, museology, etc. According to science theory, every domain has its own area of objects and of methods and processes, next to other relationships. In knowledge organization one expresses the objects by "(all kinds of) knowledge" and the methods by "organization" in the sense of creating order of the given kinds of knowledge and its activities.

Peter Ohly: ISKO's declared aims have to be seen historically and structurally. In its origin it emerged from library science cataloging. The forerunner GfKl (German Society for Classification) was founded in contrast to the DGD (German Society for Documentation) as a society with more theoretical and methodological orientation and with less stress on documentation praxis. When at least in this society the statisticians got the majority, ISKO was founded by the non-statistician part as a society with less orientation to business informatics. Thus far ISKO has still today problems to get connections to more computer-oriented neighbor fields, like knowledge management.

Furthermore ISKO has problems to claim a focus that is not already occupied by other established scientific neighbor communities, e.g., artificial intelligence, neuroscience. Thus ISKO has to define explicitly its boundaries to know with whom and how to interact and not to compete with. Such restrictions for ISKO as a society do not apply in the same determination to $\mathrm{KO}$ as a theoretical field, as a university discipline, or as a journal focus.

The main problem for ISKO as a society is that it does not attract a well-defined profession: "Knowledge organizer" or "Semantic worker" are not established professions. Accordingly the community of $\mathrm{KO}$ must be more moderate and realize that it has its main application and acceptance in library science. I would describe the focus of $\mathrm{KO}$ more general as arranging of knowledge, instead of ordering, classification or organization. How far extraction, connection, reasoning, or interpretation of knowledge should be also included in the focus of ISKO should carefully be considered and optionally be denied. Surely the economic dimension of valuable information, the scientific background of the applied field, and the sociological aspects are missing in the definition of its approaches. The latter is important for studying the acceptance of systems as well as the social dynamics of use and misuse, not at least in social software models.

Wiesław Babik: There are many definitions of knowledge organization. Although they are not always fully coherent, after summing up they define the content and the range of this notion. The main difficulty in defining knowledge organization - in my opinion - is the fact that all the definitions are based on two other notions which are very often defined only in an intuitive way: the notions of knowledge and organization. This way we produce a kind of etymological definitions. The answer for the question asked at the beginning depends on the way we understand what knowledge is and what organization is. Besides, one should always remember that the subject (topics) of knowledge organisation is composed of knowledge and information seen as raw material for knowledge, but understood from the perspective of its organization (viewpoint). This locates knowledge organization among other scientific disciplines concerned with various aspects of knowledge. Finishing I would like to express my belief that knowledge organization is a very important inter- and multidisciplinary domain, indispensable to science, education and research, which is also practiced within modern information science. 
Amos David: The way knowledge is acquired, represented, managed and exploited has changed with the connected world and the functionalities associated. I would suggest that these issues related to knowledge in the digital world should be included as an object of research.

Vera Dodebei: Although cultural approaches may be included in the semantic conceptual frame, ISKO should increase discussions on cultural aspects, considering, for example, the connected societies.

Grant Campbell: Taking off from your observation that we can create a glossary of architecture, for example, without being labeled architects, I suggested that KO's greatest strength lies in its position outside of domains and its capacity for working between them. Whereas disciplines and domains tend to focus on cultivating their own terms and their own practices and traditions and canonical texts, KO has a more itinerant role: rather like the traveler who travels from town to town, and in exchange for a meal by the fire, relays to the inhabitants stories of $\mathrm{O}^{-}$ ther places.

Dagobert Soergel: Among the ISKO membership there is a great deal of expertise in principles of knowledge organization. But most ISKO members come out of a library and bibliographic systems tradition. This presents somewhat of a barrier to bring this expertise to the much wider arena where it is applicable and where it would be beneficial. Documentary information is just a part of the information landscape. There are other applications of high importance, such as, to name a few,

- electronic health records (EHR) connected to patient treatment and medical research on the one hand and medical billing on the other hand

- scientific data

- research networking systems

- business information systems

- linked data as a format

To enable transfer of ISKO expertise into these wider application areas and the associated communities requires a re-orientation. ISKO members need to work in other areas as illustrated, for example, in the paper by Maria Lopez-Huertas. We need to invite speakers from other communities to ISKO conferences (and actively solicit contributed papers which would be subject to the review process). At the same time we need to make sure that there is a sufficient number of papers of interest to members of other communities.
Claudio Gnoli: ISKO resources that can help a more clear identification of what is covered by $\mathrm{KO}$ are 1) our journal, 2) the online $\mathrm{KO}$ literature, 3) the dictionaryglossary of $\mathrm{KO}$ that we are planning to publish on our website. Also when browsing mailing lists in order to feed the online list of coming KO-related events I often have to decide whether they are so or not, and I especially refer to topics listed in their calls for papers: I take that a $\mathrm{KO}$ event, independently from the technical means and carriers it addresses, should have to do in some way with the subject content of documents, in Buckland's broad sense of document [see his keynote at this conference] so clearly not just in libraries as feared by Soergel. I agree that developments of the semantic web should be considered and included in KO. While most current work in the semantic web is concerned with technical interoperability, KO should contribute as for conceptual interoperability (conceptual mapping, SKOS, OWL, etc.).

\subsection{What changes do you foresee in the future that will prove to be the most challenging for ISKO?}

Vera Dodebei: I see a number of questions we must address: 1) How will $\mathrm{KO}$ researchers consider the challenge of accessing memory data (traces) and heritage representations from collective knowledge in the internet? May we consider forgetting the default for memory? 2) What may be a sustainable world considering the information and knowledge society? 3) How can we keep our collective memory knowledge safe in an unstable space always in movement? To this I raise one possible answer: we must be in connection with theories from multiple fields, especially in the domain of art, history, anthropology, archeology.

Amos David: The most important challenge that I see is the temptation of being attracted by technologicallydriven research.

Ingetraut Dahlberg: If we agree that knowledge organization is a scientific discipline in its own right, we need to develop it accordingly and start with elaborating its roots. Eugen Wuester, the Austrian Terminologist in the early thirties, had paved the way by developing a series of standards for concepts (DIN 2330, etc.). I developed a new theory of the concept, published in German in the first issue of International Classification in 1974 and in English in the volume of the FID/CR Conference in Bombay in 1975. In later publications I extended it further, calling a concept a unit of knowledge. According to this theory a concept needs to be analyzed into its characteristics; by finding the same or similarly expressed characteristics in different concepts, true systems of concepts 
can be created into the two hierarchical, and the complementary and functional relationships. This I have shown in many publications already in the seventies, the last one in German in 2008 and in English somewhat later in KO 2009. It is also contained in an abridged way in the article on "A Systematic New Lexicon of All Knowledge Fields" with the theories and principles of the ICC (KO 2012). I would like to add that I learned very much from the mathematician and librarian S. R. Ranganathan and consider him still fundamental for all of our students.

Peter Ohly: KO goes back to known principles of concept formation, as applied in the developing of indexing systems. But instead of seeing it mostly in the field of cataloging it must be more open to realize that there are new applications, new knowledge sources and quite other applications, like virtual knowledge generation, mobile devices, decision making, evaluation indexes. We often speak of literacy and think of KO literacy for the users of other disciplines. Instead we should realize that we have permanent need for understanding new upcoming techniques and thinking in neighboring fields. Openness can be reached by tutorials, workshops, and co-operation that broaden our understanding and applicability of neighboring disciplines, specialized areas, and other cultures.

Grant Campbell: Two challenges will confront $\mathrm{KO}$ in the future, as it will other disciplines: 1) The problem of cultural and community differences: Canada is currently undergoing considerable stress from its earlier catastrophic treatment of indigenous peoples, in particular around education and, by implication, organizations and structures of knowledge. KO will be urgently needed by many countries and cultures that are trying to negotiate the demands of different cultures, and trying to step outside the usual "one size fits all" approach to enforcing uniformity. 2) The need to develop sustainable economies and cultures will require major changes in KO: changes that will enable all of us to think more easily and clearly in terms of sustainability. ISKO 2016's announced theme couldn't be more appropriate or timely.

Jill McTavish: One challenge I see for ISKO's future is how to better incorporate new, different, and upcoming voices. It's much along the line of what Grant Campbell and José Augusto Chaves Guimarães have said. If one were to examine the discourse of our conference there would be only a few voices that dominated throughout. Why don't the students feel comfortable commenting? Why are there only a handful of people that felt comfortable commenting throughout the conference? How can those that feel comfortable talking try to create spaces for others to speak, and how can ISKO structure different voices into its programming? For example, a student representative could have been on both of the panels offered at ISKO 2014. ISKO 2016 could also offer partial conference scholarships to new students-perhaps through an essay contest, lottery, or something.

Wiesław Babik: I am absolutely convinced that today we should promote a network approach to knowledge organization, both in its theoretical and conceptual dimensions as well as in practical ISKO activities. For the last 25 years we have been able to observe an explicit tendency toward automation, globalization and socialization of information and knowledge creation processes, including knowledge organization and ISKO activities. ISKO has noticed those changes and has been trying to take them into consideration in its deliberations and activities. But there are also some threats that should be noticed. In such a situation, both ISKO and KO should be more human-oriented and sustainable development driven, and these should be the most important challenges for the next 25 years. Knowledge organization and ISKO cannot forget about human beings, which seems to be quite common in the present world.

\subsection{What is your ideal picture of what the ISKO of the future could be? How do we get there?}

Amos David: ISKO has a sound foundation. This should be maintained and reinforced. ISKO conferences constitute an excellent forum for scientific exchanges and Knowledge Organization has a very good reputation. To maintain its level of recognition, the community should remain focused on scientific objects rather than technology-dependent issues.

Laura Ridenour: I need to make a disclaimer: I don't have a library background, and I hope I won't be lynched for what I am about to say. I would like to suggest that knowledge organization needs to consider an open access model of publication to provide access for people who may be interested in $\mathrm{KO}$, but are not part of the community. My reasoning is that Knowledge Organization (the journal) is not widely accessible; knowledge organization literature is both difficult to locate and misindexed in databases such as LISTA, usually placed under knowledge management; (related to my first point) that in order to be relevant, we must contribute to scientific literature in a forum in which more people are able to access our bodies of work; and we need to collaborate with individuals in other research specialties. 
Wiesław Babik: In my opinion both ISKO and Knowledge Organization will benefit from the implementation of the idea of information and knowledge society, because this process demands high quality information and knowledge. Knowledge organization can help to regain control over a chaotic information world, both literally and metaphorically, especially when supported by knowledge and information ecology. This new domain has already found its place in academic teaching within study programmes connected with information and in research. In ISKO activities one can separate three basic levels: international, national and local. All of them should be developed in a balanced way. This should be better represented by the ISKO structure. The structure of the Polish chapter could be seen as an interesting example.

Peter Ohly: ISKO should become a virtual institute where from everywhere one can be connected with specialists in $\mathrm{KO}$ and get their advice. This should not only be performed in a KO-pedia style but also as an e-science, where projects are performed virtually with scientists, coming as well from other disciplines. KO managers should not only know what is the best $\mathrm{KO}$ but as well be able to explain and elaborate the differences, strengths and weakness of special KO approaches in special applications.

Dagobert Soergel: Ideally, ISKO would develop into a society that covers $\mathrm{KO}$ issues in a wide range of applications, with keen attention to common principles, and that attracts people focusing on $\mathrm{KO}$ from many communities, serving as a common meeting point for the transfer of basic knowledge and of reusable modules in the development of KO systems. Along similar lines, ISKO should get involved in formulating information literacy standards so these standards incorporate not just surface skills in searching for information but deeper understanding of principles of knowledge organization that will make people much better searchers. Within $\mathrm{KO}$ courses in library and information science programs, we need to make students keenly aware of the wide range of $\mathrm{KO}$ applications, the areas, and widely used systems, such as
- CYC Ontology

- WordNet

- Gene Ontology (GO)

- SnoMed

- ICD-10 (The International Statistical Classification of Diseases and Related Health Problems, tenth revision)

- CPT (Physicians' Current Procedural Terminology. CPT 2003)

- NAICS (North American Industrial Classification System)

- HS (Harmonized Commodity Description and Coding System. World Customs Organization)

- Bloom: A taxonomy for learning, teaching, and assessing: A revision of Bloom's taxonomy of educational objectives

- AAT (The Art and Architecture Thesaurus)

- Iconclass

This extension of the range should also be pursued for the journal KO. A wider range and higher quality of submissions would ideally elevate the recognition and prestige of the journal, increase readership (readers from many different communities) and elevate $K O$ into a firsttier journal. Finally, it would be useful to create a list of associations, conferences, and separate listservs that deal with $\mathrm{KO}$ and also repositories for KOS. Some I can think of are:

- ASIST SIG-CR: Organizes a one-day workshop at ASIST Annual Meeting

- IAOA (International Applied Ontology Organization): Organizes FOIS (Formal Ontology for Information Systems) conference

- NKOS

- Ontolog-forum: Organizes the yearly Ontology Summit

- Yearly ontology matching workshop

- ICBO (International Conference on Biomedical Ontologies): every other year. 